\title{
Numerical Simulation of Sudden Contraction Flow for Viscoelastic Fluids with the Lattice Boltzmann Method
}

\author{
Qing YAO ${ }^{1, a}$, Wang-Jun ZHOU ${ }^{1}$, Yong $\mathrm{LI}^{1}$ and $\mathrm{Lu}-\mathrm{Wu} \mathrm{HE}{ }^{1}$, * \\ ${ }^{1}$ School of Mechanical and Power Engineering, East China University of Science and Technology, \\ Shanghai, 200237, China \\ ayaoqing@mail.ecust.edu.cn
}

Keywords: LBM, Viscoelastic fluid, Sudden contraction flow, Reynolds numbers Re, Viscosity $v_{s}$, Weissenberg numbers Wi.

\begin{abstract}
In this paper, based on the lattice Boltzmann method (LBM) and the Oldroyd-B model, the decoupled solving method for the incompressible Navier-Stokes equation and advectiondiffusion constitutive equation and the boundary processing formats are given. The flow of viscoelastic fluid in the planar 3:1 contraction channel is simulated. For different Reynolds numbers Re, Weissenberg numbers Wi and viscosity $v_{s}$, the streamline patterns, the positions of vortex center and the length of vortex are obtained, and the influences of these parameters on flow behavior are discussed. The numerical results also present that the decoupled solving method is feasible and the LBM has fine accuracy and stability.
\end{abstract}

\section{Introduction}

Lattice Boltzmann method (LBM) is a mesoscopic model between the fluid microscopic molecular dynamics model and macroscopic model, which is different from the macro level of the traditional numerical simulation methods (such as finite difference method, finite volume method, etc.). On the contrary LBM is based on the collision and migration of fluid particle to describe the flow phenomenon, and treat the fluid as abstract particles. Due to its clear evolution process, strong parallel performance and suitable for large-scale numerical calculation, LBM has become a powerful tool to simulate a variety of physical phenomena, and showed a broad prospect in the engineering application $[1,2]$.

Viscoelastic fluid widely exists in the plastics industry, chemical industry, petroleum industry, food industry and biological engineering, etc. Different from the Newtonian fluid, this class of fluids is characterized by the fact that the deformation of an element of fluid induced by a stress does depend not only on the strain itself but also on the history of the deformation (memory effect). Common viscoelastic constitutive model [3] is divided into two major categories of differential and integral. Differential type constitutive model mainly contains Maxwell, Oldroyd-B and PTT, and integral type constitutive model mainly contains $\mathrm{K}$ - BKZ, etc.

In many engineering applications, the numerical simulation has become an effective technique to predict the behavior of viscoelastic fluid. In 1997, Qian and Deng [4] had used the lattice Boltzmann method to simulate the viscoelastic fluid flow. In their work, the equilibrium distribution function had been changed in order to reflect the elastic effect. But this method has only considered the unilateral elastic effect, and not really brought in the constitutive equation. Giraud [5] and Lallemand [6] established the lattice Boltzmann model of Jeffreys fluid later by introducing Jeffreys constitutive equation. But they treated the stress tensor and strain tensor of Jeffreys constitutive model as a linear relationship, ignoring the memory of stress tensor, so they hadn't took some important elastic effect into account. Because Maxwell model is simple related to others and the stress tensor could be expressed in terms of the integral, so in 2002, Ispolatov [7] established Maxwell fluid of the lattice Boltzmann model by introducing Maxwell external forces. A preliminary analysis shows that the model can accurately simulate the viscoelastic behavior of viscoelastic fluid, but limited by the stability, only a small relaxation time could be computered. This work also ignored some important elastic effect as Giraud [5] did, but the model was 
successfully used to simulate the bubble motion in the shear flow [8,9]. In 2010, Malaspinas [10] introduced dual distribution function to simulate Oldroyd-B fluid. In this work, the configuration tensor was used instead of stress tensor [11,12] in the Oldroyd-B constitutive model, and the convection diffusion lattice Boltzmann model [13] was used to solved the configuration tensor component, then the stress tensor could be obtained through the configuration tensor.

The outline of this paper is the following. Section 2 describes the Oldroyd-B constitutive model. Section 3 discusses the decoupled solving methods for incompressible Navier-Stokes equation and advection-diffusion constitutive equation and gives the boundary processing format. Section 4 numerically simulates viscoelastic fluid flow in the two-dimensional 3:1 contraction flow channel, and obtains the streamline distribution, the positions of vortex center and length of vortex with different Reynolds numbers Re, Weissenberg numbers Wi and viscosity $v_{s}$, and discusses the influences of these parameters for the streamline distribution.

\section{Constitutive Model}

In simulating the polymer processing, differential type of PTT constitutive model can more accurately describe the viscoelastic rheological behavior of polymer fluid. The stress tensor is decomposed into pure shear $\sigma_{s}$ and polymer viscoelastic $\Pi$ as follows :

$$
\sigma=\sigma_{s}+\Pi
$$

For the Oldroyd-B model (a kind of PTT model), the viscoelastic deviatoric stress tensor is given by

$$
\boldsymbol{\Pi}=\frac{\mu_{p}}{\lambda}(\boldsymbol{A}-\boldsymbol{I})
$$

where $\lambda$ is polymer relaxation time, and $\boldsymbol{A}$ is conformation tensor (a statistical indicator of the orientation of the polymer molecules). Then the constitutive equation, written in terms of the conformation tensor $\boldsymbol{A}$, reads

$$
\frac{d \boldsymbol{A}}{d t}=-\frac{1}{\lambda}(\boldsymbol{A}-\boldsymbol{I})+\boldsymbol{A} \cdot \nabla \boldsymbol{u}+(\nabla \boldsymbol{u})^{T} \cdot \boldsymbol{A}
$$

where $d / d t=\partial \mathrm{t}+(\mathbf{u} \cdot \nabla)$ is material derivative, and $\boldsymbol{u}$ is fluid velocity.

\section{Numerical Model}

Classic lattice Boltzmann method is used to solve incompressible Navier-Stokes equation, in which the viscoelastic stress tensor $\Pi$ given by solving the constitutive equation can be as a known quantity here. So the lattice Boltzmann-BGK equation with second order precision [10] is given by

$$
\begin{aligned}
\bar{f}_{i}\left(\boldsymbol{x}+\boldsymbol{e}_{i}, t+1\right)-\bar{f}_{i}(\boldsymbol{x}, t)= & -\frac{1}{\bar{\tau}}\left(\bar{f}_{i}(\boldsymbol{x}, t)-f_{i}^{(0)}(\boldsymbol{x}, t)\right) \\
& +\left(1-\frac{1}{2 \bar{\tau}}\right) F_{i}
\end{aligned}
$$

where the $\boldsymbol{e}_{i}$ s are the discrete microscopic velocities in the $i$ th direction and $\bar{\tau}$ is the relaxation time. The action of forces $F_{i}$ and equilibrium distribution function $f_{i}^{(0)}(\boldsymbol{x}, t)$ is as follows:

$$
\begin{array}{r}
F_{i}=\omega_{i} \rho\left(\frac{\boldsymbol{e}_{i}-\boldsymbol{u}}{c_{s}^{2}}+\frac{\left(\boldsymbol{e}_{i} \cdot \boldsymbol{u}\right) \boldsymbol{e}_{i}}{c_{s}^{\mathbf{4}}}\right) \cdot \boldsymbol{g} \\
f_{i}^{(0)}=\omega_{i} \rho\left(1+\frac{\boldsymbol{e}_{i} \cdot \boldsymbol{u}}{c_{s}^{2}}+\frac{1}{2 c_{s}^{4}} \boldsymbol{Q}_{i}: \boldsymbol{u} \boldsymbol{u}\right)
\end{array}
$$

with $\boldsymbol{Q}_{i}=\boldsymbol{e}_{i} \boldsymbol{e}_{i}-c_{s}^{2} \boldsymbol{I}$ whereas the colon ":" sign denotes full index contraction. The symbols $\omega_{i}$ and $c_{s}$ are respectively the weights and the sound speed of the lattice. The quantity $\boldsymbol{u} u$ represents the tensor product of the vector $\boldsymbol{u}$ by itself and $\rho \boldsymbol{g}$ represents the force density. The distribution function $f_{i}$ can 
use the D2Q9 model, as shown in Fig.1. The macro density $\rho$, velocity $\boldsymbol{u}$ and pressure $p$ respectively are given by

$$
\rho=\sum_{i=0}^{q-1} \bar{f}_{i}, \boldsymbol{u}=\frac{1}{\rho} \sum_{i=0}^{q-1} \boldsymbol{e}_{i} \bar{f}_{i}+\frac{\boldsymbol{g}}{2}, p=c_{s}^{2} \rho
$$

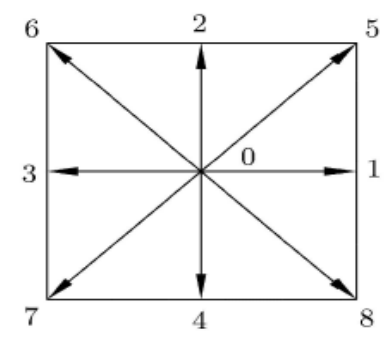

Figure 1. The D2Q9 lattice model

In order to solve configuration tensor $\boldsymbol{A}$, Denniston [14] and Marenduzzo [15,16] Et al. adopt a revised lattice Boltzmann model [17] to obtain the distribution function $h_{i \alpha \beta}$ by calculating the advection-diffusion equation, thereby the configuration tensor $A_{\alpha \beta}$ of each component can be got to discrete lattice Boltzmann equation :

$$
\begin{aligned}
\bar{h}_{i \alpha \beta}\left(\boldsymbol{x}+\boldsymbol{e}_{i}, t+1\right)-\bar{h}_{i \alpha \beta}(\boldsymbol{x}, t)= & -\frac{1}{\bar{\varphi}}\left(\bar{h}_{i \alpha \beta}(\boldsymbol{x}, t)-\bar{h}_{i \alpha \beta}^{(0)}\left(\boldsymbol{A}_{\alpha \beta}, \boldsymbol{u}\right)\right) \\
& +\left(1-\frac{1}{2 \bar{\varphi}}\right) \frac{\varsigma_{\alpha \beta}}{\boldsymbol{A}_{\alpha \beta}} h_{i \alpha \beta}^{(0)}\left(\boldsymbol{A}_{\alpha \beta}, \boldsymbol{u}\right)
\end{aligned}
$$

where $\bar{\varphi}$ is the relaxation time, and $\bar{h}_{i \alpha \beta}$ is distribution function of configuration tensor. The source term $\varsigma_{\alpha \beta}$, equilibrium distribution function $h_{i \alpha \beta}^{(0)}$ and the relationship between distribution function $\bar{h}_{i \alpha \beta}$ and configuration tensor $\boldsymbol{A}$ show as

$$
\begin{gathered}
\boldsymbol{\varsigma}=-\frac{1}{\lambda}(\boldsymbol{A}-\boldsymbol{I})+\boldsymbol{A} \cdot(\nabla \boldsymbol{u})+(\nabla \boldsymbol{u})^{T} \cdot \boldsymbol{A} \\
h_{i \alpha \beta}^{(0)}=\omega_{i} \boldsymbol{A}_{\alpha \beta}\left(1+\frac{\boldsymbol{e}_{i} \cdot \boldsymbol{u}}{c_{l}^{2}}\right), \boldsymbol{A}_{\alpha \beta}=\overline{\boldsymbol{A}}_{\alpha \beta}+\frac{\boldsymbol{\varsigma}_{\alpha \beta}}{2}, \overline{\boldsymbol{A}}_{\alpha \beta}=\sum_{i=0}^{q-1} \bar{h}_{i \alpha \beta}
\end{gathered}
$$

By using the Chapman-Enskog expansion method, we can get the following equation :

$$
\frac{d \boldsymbol{A}_{\alpha \beta}}{d t}=\boldsymbol{\varsigma}_{\alpha \beta}+\kappa \nabla^{2} \boldsymbol{A}_{\alpha \beta}+\frac{\boldsymbol{\kappa}}{c_{l}^{2}} \nabla \cdot\left(\boldsymbol{A}_{\alpha \beta} \partial_{t} \boldsymbol{u}-\boldsymbol{u} \nabla \cdot\left(\boldsymbol{A}_{\alpha \beta} \boldsymbol{u}\right)\right)
$$

where $\kappa=c_{l}^{2}(\bar{\varphi}-1 / 2)$

It has been proven in theory that there can be a diffusion term in the constitutive equation [18]. Research shows that the constitutive equation introduced in diffusion coefficient $\kappa$ can increase the numerical simulation stability $[19,20]$, but diffusion coefficient in practice should be very small. Advection diffusion equation choose D2Q5 model in simulation, as shown in Fig.2.

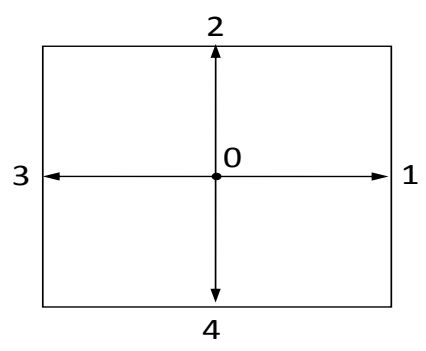

Figure 2. The D2Q5 lattice model 
In numerical calculation, the alternating iteration method have been adopted for Eq. (4) and (7). On the one hand, with the Navier-Stokes scheme, we compute the velocity and its gradients and the source term in Eq. (9). On the other hand, the advection-diffusion scheme enables one to estimate the conformation tensor, which is transformed into the viscoelastic stress tensor $\Pi$ by using Eq. (2).

In this paper, the distribution function $f$ uses the D2Q9 model and boundary treatment uses nonequilibrium extrapolation format. The the distribution function $h$ uses D2Q5 model, and boundary treatment here adopts a same approach as in [10].

\section{The Results of Numerical Simulation and Analysis}

Using the above method, the numerical simulation of viscoelastic fluid flow behavior in planar contraction flow channel is discussed as follow. The geometric model of the channel is shown in Fig.3, and the symbols $h, H, L_{1}, L_{2}, L_{r}$ are respectively the height of inlet, the height of outlet, the entrance length, the contract length, and vortex flow length of wall side. In the following numerical simulation, $h=120, h: H=3: 1, L_{1}=120, L_{2}=120 . v_{s}, v_{p}$ are respectively kinematic viscosity of the solution and polymer, and the viscosity ratio $\mathrm{R}_{v}=v_{s} / v_{p}=1 / 8$. In the actual calculation model, the expression of Reynolds number Re and Weissenberg numbers Wi can be obtained: $\mathrm{Wi}=2 \lambda u_{\max } / \mathrm{h}$, $\operatorname{Re}=u_{\max } h / 2 v_{s}$, where $u_{\max }$ is the maximum speed of entry.

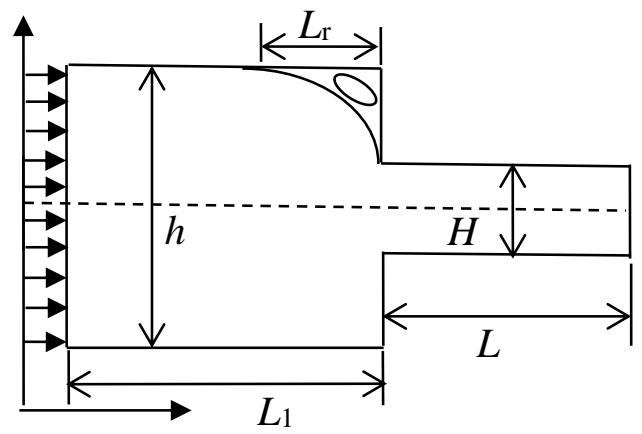

Figure 3. Geometry of the channel

In order to study the flow law of the viscoelastic fluid in a two-dimensional contraction flow channel, this paper set a total of 12 groups of different parameters. We have divided into three cases to study respectively the influence of the parameters Re, $v_{s}$ and Wi. Table.1 list the parameter values used in numerical simulation:

Table 1. The parameter list of 2D contraction flow.

\begin{tabular}{|l|l|l|l|l|}
\hline & $\operatorname{Re}$ & $\mathrm{Wi}$ & $v_{s}$ & $u_{\max }$ \\
\hline & 1.0 & & & 0.009 \\
Case 1 & 2.0 & & & 0.018 \\
& 3.0 & 0.10 & 0.06 & 0.027 \\
& 4.0 & & & 0.036 \\
\hline & & & 0.06 & 0.009 \\
Case 2 & 1.0 & 0.10 & 0.09 & 0.015 \\
& & & 0.12 & 0.0225 \\
& & & 0.15 & 0.030 \\
\hline & & 0.10 & & \\
Case 3 & 1.0 & 0.20 & 0.06 & 0.009 \\
& & 0.30 & & \\
\hline
\end{tabular}


Fig.4 shows the influences of different Re on the streamlines in contraction flow with $v_{s}=0.06$, $\mathrm{Wi}=0.1$. Table. 2 gives the coordinate value $(x, y)$ of vortex core and the length of backflow $L_{r}$ with different Re numbers.

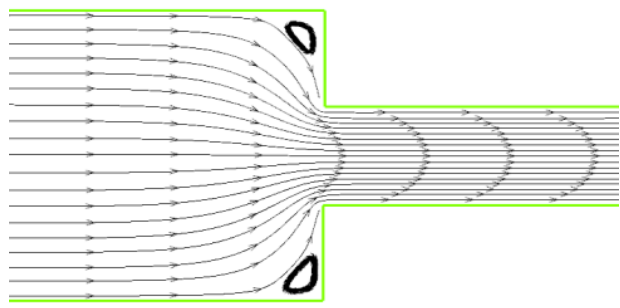

(a) $\operatorname{Re}=1.0$

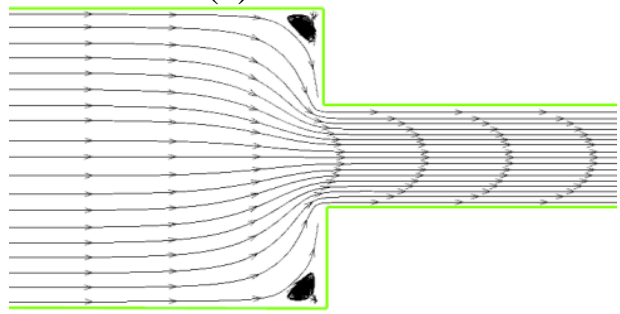

(c) $\operatorname{Re}=3.0$

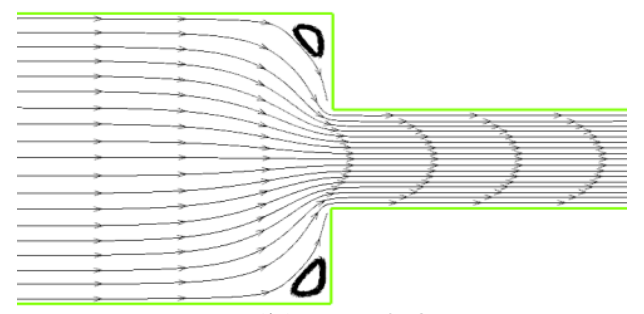

(b) $\operatorname{Re}=2.0$

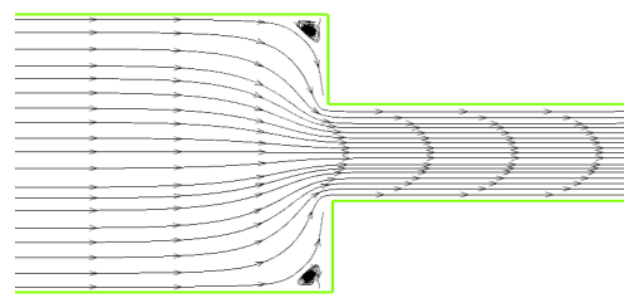

(d) $\operatorname{Re}=4.0$

Figure 4. Streamlines in contraction flow for different $\operatorname{Re}\left(v_{s}=0.06, \mathrm{Wi}=0.1\right)$

Table 2. The coordinate value $(x, y)$ and $L_{r}$ for different $\operatorname{Re}$

\begin{tabular}{|l|l|l|l|l|}
\hline $\operatorname{Re}$ & 1.0 & 2.0 & 3.0 & 4.0 \\
\hline$x$ & 113.79 & 114.19 & 114.84 & 115.43 \\
\hline$y$ & 111.82 & 113.70 & 114.85 & 115.69 \\
\hline$L_{r}$ & 15.25 & 13.61 & 12.50 & 10.75 \\
\hline
\end{tabular}

It can be seen from Table.2, for $\mathrm{Wi}=0.1, v_{s}=0.06$, with the increase of Re, that $x$ and $y$ coordinate of vortex core increases gradually for the upper part, which means that vortex core is more and more close to the respective corner, and that the length of backflow $L_{r}$ decreases, and the corresponding flow area decreased gradually too.

Fig.5 displays the influences of different $v_{s}$ on the streamlines in contraction flow with $\mathrm{Re}=1.0$, $\mathrm{Wi}=0.1$. Table. 3 shows the coordinate value $(x, y)$ of vortex core and the length of backflow $L r$ with different $v_{s}$.

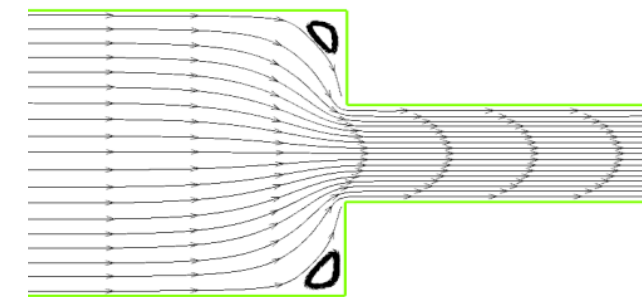

(a) $v_{s}=0.06$

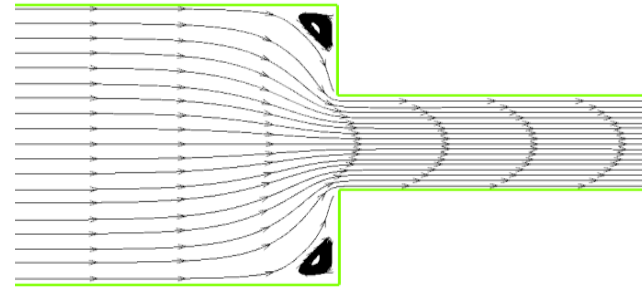

(c) $v_{s}=0.12$

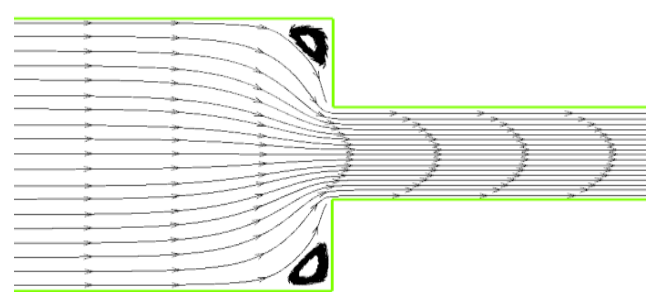

(b) $v_{s}=0.09$

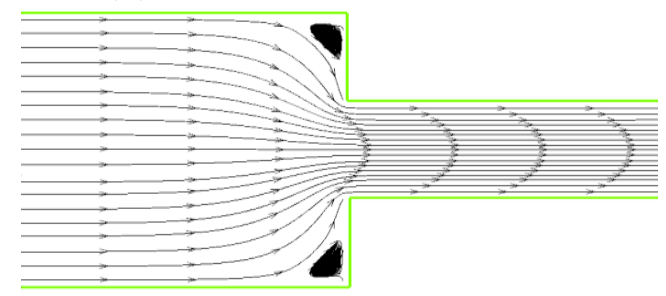

(d) $v_{s}=0.15$

Figure 5. Streamlines in contraction flow for different $v_{s}(\mathrm{Re}=1.0, \mathrm{Wi}=0.1)$ 
Table 3. The coordinate value $(x, y)$ and $L_{r}$ for different $v_{s}$

\begin{tabular}{|l|l|l|l|l|}
\hline$\nu_{\mathrm{S}}$ & 0.06 & 0.09 & 0.12 & 0.15 \\
\hline$x$ & 113.79 & 114.05 & 114.30 & 114.48 \\
\hline$y$ & 111.82 & 111.64 & 111.47 & 111.40 \\
\hline$L_{r}$ & 15.25 & 16.64 & 15.68 & 15.74 \\
\hline
\end{tabular}

It can be seen from the Table. 3 , for $\mathrm{Wi}=0.1, \mathrm{Re}=1.0$, with the increase of $v_{s}$, that $x$ coordinate of vortex core increases for the upper part, but $y$ coordinate decreases gradually, which means that vortex core is more and more close to the respective contraction entrance, and that the length of backflow $L_{r}$ first increases and then decreases.

Fig.6 shows the influences of different Wi on the streamlines in contraction flow with $\mathrm{Re}=1.0, v_{s}$ $=0.06$. Table 4 gives the coordinate value $(x, y)$ of vortex core and the length of backflow $L_{r}$ with different Wi numbers.

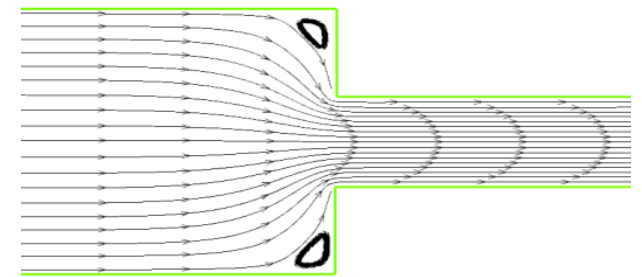

(a) $\mathrm{Wi}=0.10$

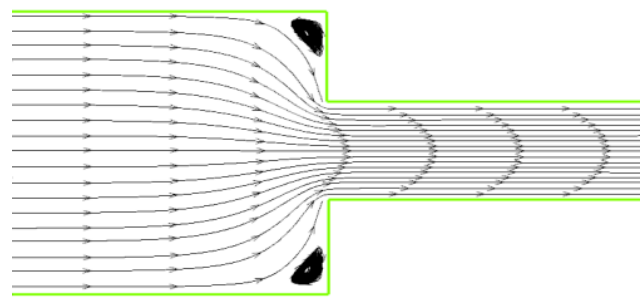

(c) $\mathrm{Wi}=0.20$

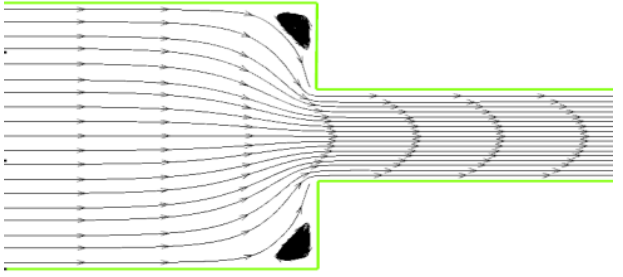

(b) $\mathrm{Wi}=0.15$

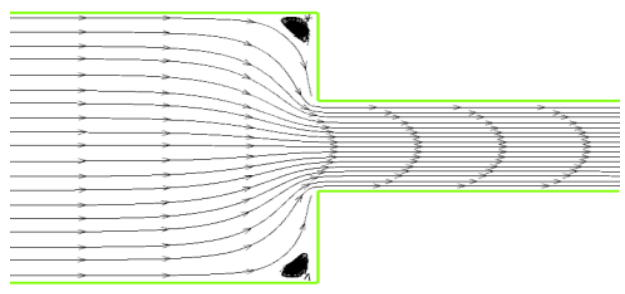

(d) $\mathrm{Wi}=0.25$

Figure 6. Streamlines in contraction flow for different $\mathrm{Wi}\left(\mathrm{Re}=1.0, v_{s}=0.06\right)$

Table 4. The coordinate value $(x, y)$ and $L r$ for different $\mathrm{Wi}$

\begin{tabular}{|l|l|l|l|l|}
\hline $\mathrm{Wi}$ & 0.10 & 0.20 & 0.30 & 0.40 \\
\hline$x$ & 113.78 & 114.41 & 114.66 & 114.89 \\
\hline$y$ & 111.82 & 111.60 & 111.75 & 114.81 \\
\hline$L_{r}$ & 15.25 & 16.24 & 15.64 & 12.67 \\
\hline
\end{tabular}

It can be seen from the Table.4, for $\mathrm{Re}=1.0, v_{s}=0.06$, with the increase of $\mathrm{Wi}$, that $x$ coordinate of vortex core increases gradually for the upper part, and that the $y$ coordinate first decreases and then increases, but length of backflow $L_{r}$ first increases and then decreases.

\section{Conclusion}

In this paper, we apply the lattice Boltzmann method combining Oldroyd-B model to discuss the decoupled solving methods for the incompressible Navier-Stokes equation and advection diffusion constitutive equation and give the boundary processing format. Finally we implement the numerical simulation for viscoelastic fluid flow in the two-dimensional 3:1 contraction flow with different Reynolds number Re, viscosity $v_{\mathrm{s}}$ and Weissenberg numbers Wi. We also obtain the streamline distribution, the location of vortex core and the size of the vortices in each condition, and discuss the influence of the these parameters on streamline distribution. The simulation results in this paper 
show that the model and processing method have good accuracy and stability, which has laid a good foundation for later research work (such as free surface, multiphase fluid).

\section{Acknowledgement}

This work is supported by National Natural Science Foundation of China (Grant NO.11372111)

\section{References}

1. Yaling he, Yong Wang, Qing Li, Theory and applications of lattice boltzmann method[M], beijing, Science Press.(in chinese)

2. Zhaoli guo, Chuguang Zheng,Theory and applications of lattice boltzmann method[M], beijing, Science Press.(in chinese)

3. Hesheng Liu, Zhigang Tu, Honghuai Xiong, Viscoelastic constitutive equation of polymer melts, Polymer Materials Science and Engineering . 18(1)22-25(2002).( in chinese)

4. Y.-H. Qian, Y.-F. Deng. Phys. Rev. Lett. 79 (14) 2742-2745 (1997)

5. L. Giraud, D. d'Humieres, P. Lallemand, Europhys. Lett. 42 (6) 625-630 (1998)

6. P. Lallemand, D. d'Humieres, L.-S. Luo, R. Rubinstein. Phys. Rev. E 67 (2) 021203 (2003)

7. Ispolatov, M. Grant. Phys. Rev. E 65 (5) 056704 (2002)

8. M. Yoshino, Y. Toriumi, M. Arai, J. CST 2(2) 330-340 (2008)

9. X. Frank, H. Z. Li. Phys. Rev. E 74056307 (2006)

10. O.Malaspinas, N. Fiétier, M. Deville, J. NNFM 165 1637-1653 (2010)

11. J. Su, J. Ouyang, X. D. Wang, and B. X. Yang. Phys. Rev. E 88053304 (2013)

12. J. Su, J. Ouyang, X. D. Wang, and B. X. Yang, J. NNFM 194 42-59 (2013)

13. Z. Guo, B. Shi, C. Zheng, Int. J. NMF 39 325-342 (2002)

14. C. Denniston, E. Orlandini, J. M.Yeomans, J. Phys. Rev. E 63 (5) 056702 (2001)

15. D. Marenduzzo, E. Orlandini, M.E. Cates, J. M. Yeomans, J. NNFM 149 56-62 (2008)

16. D. Marenduzzo, E. Orlandini, M.E. Cates, J. M. Yeomans, J. Phys. Rev. E 76031921 (2007)

17. Z. Guo, B. Shi, C. Zheng, J. NMF 39 325-342 (2002)

18. A.W. El-Kareh, L. G. Leal, J. NNFM 33 (3) 257-287 1989

19. R. Sureshkumar, A. Beris, J. NNFM 60 53-80 (1995)

20. K. Housiadas, A. Beris, J. NNFM 122 243-262 (2004) 